\title{
Full Mouth Rehabilitation of a 4-Year-Old Child with Severe Early Childhood Caries: A Case Report
}

\author{
Lakshmi Thribhuvanan ${ }^{1}$ MS Saravanakumar ${ }^{1}$ Anjana G \\ 'Department of Pedodontics and Preventive Dentistry, Royal Dental \\ College, Palakkad Kerala, India
}

\begin{abstract}
Address for correspondence: Lakshmi Thribhuvanan, MDS, Department of Pedodontics and Preventive Dentistry, Royal Dental College, Iron Hills, Chalissery, Palakkad 679536, Kerala, India

E-mail: lakshmithribhuvanan91@gmail.com
\end{abstract}

\begin{abstract}
Early Childhood Caries (ECC) is defined as the presence of one or more carious lesions, which are either cavitated or non cavitated in children up to five years of age. The primordial risk factors leading to the development of Early Childhood Caries include prolonged retention of bacterial plaque, decreased oral hygiene maintenance and frequent consumption of sugary syrups and beverages. Hence, ECC has a substantial influence on the child's lifestyle, which results in the child experiencing severe pain, premature loss of teeth, improper nourishment, difficulty in mastication, and delay in attaining normal milestones with regard to normal growth and development child both physically and psychologically. This paper presents a case report of a 4-year-old child patient who is diagnosed with Early Childhood Caries and explains in detail regarding the rehabilitation of the child's mouth with caries prevention strategies along with restorative and rehabilitative procedures with special consideration on modification of child's feeding habits and practice of good oral hygiene measures beneficial for the child.
\end{abstract}

Keywords: Early Childhood Caries, full mouth rehabilitation, oral hygiene measures

\section{Introduction}

Early Childhood Caries was previously known as baby bottle caries, nursing bottle caries, or baby bottle tooth decay.[1] Nursing decay is a relatively new term that is used nowadays for defining caries occurring in infants. [2] The greatest challenge of a pediatric dentists is the esthetic and functional rehabilitation of toddlers with Early Childhood Caries.

The factors associated with ECC are fermentable carbohydrates (substrate); cariogenic microorganisms, the frequency of intake of refined sugars, tooth enamel defects such as enamel hypoplasia; and the socioeco- nomic state of the caregiver.[3] A new pathogen, Scardovia wiggsiae, has been associated with severe early childhood caries. Streptococcus mutans and S. wiggsiae in dental plaque metabolizes the available sugars to produce reactive acids, resulting in fall of intraoral $\mathrm{pH}$ thereby resulting in subsequent loss of minerals from tooth surface and finally culminating to dental caries. [4] Prolonged $\mathrm{pH}$ drop, and the total net loss of minerals lead to a weakening eventually resulting in the breakdown of the tooth surface. Severe ECC, when left untreated, is recognized as part of the more general phenomenon of child neglect which will have debilitating effects on children.[5]

How to cite this article: Thribhuvanan L, Saravanakumar MS, Anjana G. Full Mouth Rehabilitation of a 4-Year-Old Child with Severe Early Childhood Caries: A Case Report. J Pediatr Dent 2021;7(3):00-00 


\section{Case Presentation}

A 4-year-old boy reported with the chief complaint of decayed upper front teeth to the department. It happened to be the child's first dental visit. Following which familiarization was done with the new environment of the dental clinic and clinic personnel. Subsequently, the child showed a Frankl behavior with a positive rating. Intraoral examination revealed abscesses on 51 and 61 and carious 52, 62 54, 64, 75, 74 and 85 . The crowns of the anterior maxillary teeth were grossly destroyed (Figs. 1-3).

Radiographs were advised for 51, 52, 61,62,74,75, and 85 (Fig. 4). The radiological diagnosis stated that there was pulpal involvement of $51,52,61,62,75$, and 85 , dentinal carious lesions in 54, 64, and 74. Medical history was non-contributory. Also, there was no significant history of any habit or bruxism. Following this

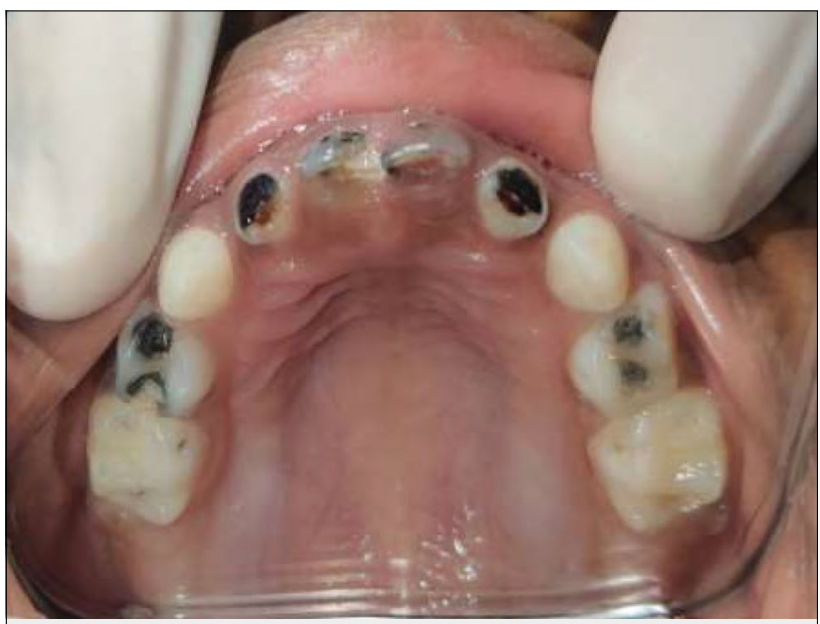

Figure 1. Preoperative photographs showing grossly Decayed 51, $52,61,62$ and carious 54,64

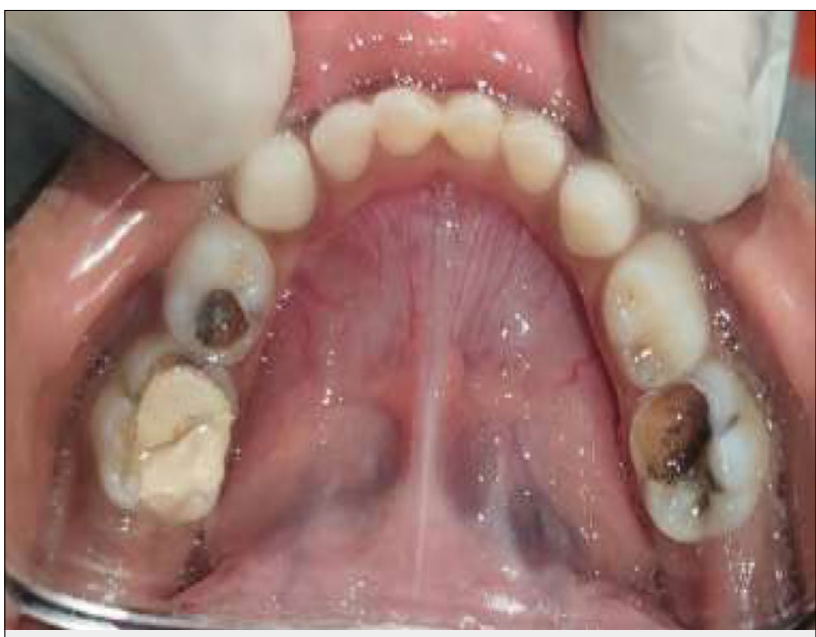

Figure 2. Preoperative photographs showing carious 74, 75 and 85

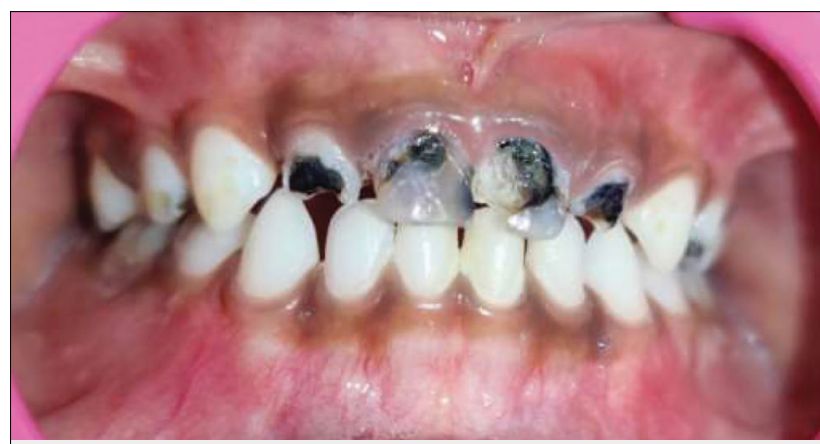

Figure 3. Preoperative photographs showing grossly decayed maxillary anterior teeth
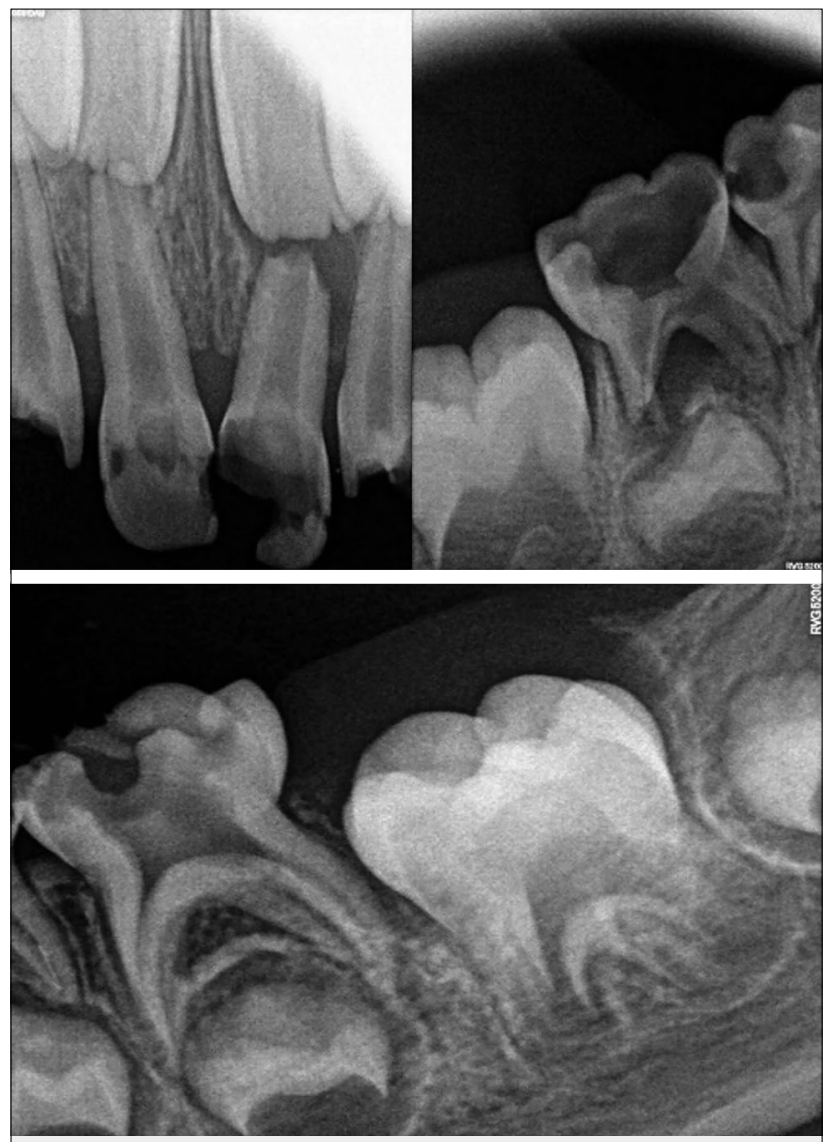

Figure 4. Preoperative radiographs showing pulpal involvement in $51,5261,62,75$ and 85

treatment plan was formulated. A written consent was obtained from parents which explained the treatment plan and expected costs for the suggested plan as well as disclosure for publishing this case report.

Pulpectomy was planned for 51, 52, 61 and 62 followed by composite restorations for the crowns (strip crowns). After the successful completion of pulpectomy with Metapex (Metapex, Meta Biomed, Korea) (calcium hydroxide-iodoform paste) obturation technique followed by GIC restoration on 51, 52, 61 and 62 (Fig. 5) the 

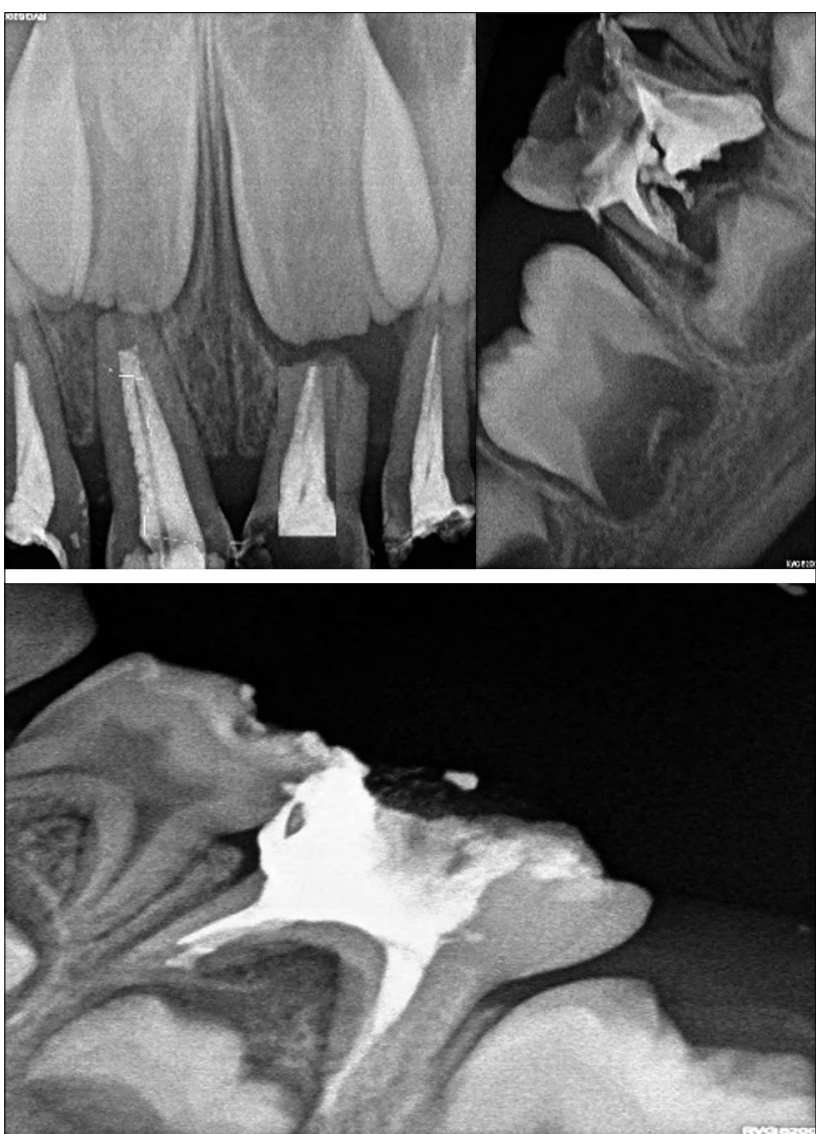

Figure 5. Postoperative radiographs showing pulp therapy in 51, $52,61,62,75$ and 85

child was recalled the following week for strip crown placement. After the removal of the GIC layer strip crowns were placed on the dental remnants in order to verify their correct adaptation to the tooth surface. Subsequently, conditioning was performed with $37 \%$ phosphoric acid (Ultra Etch-Ultradent, Ultradent Products Inc., South Jordan, UT, USA) for 15 seconds in the dentin and 30 seconds in the enamel and delicate drying with absorbent paper. The adhesive system used was the Adper Scotchbond (3M ESPE, St. Paul, MN, USA) applied according to the manufacturer's recommendations. Soon after, the strip crowns were filled with Filtek Z350 XT WD composite resin (3M ESPE, St. Paul, $\mathrm{MN}, \mathrm{USA}$ ) in color (A1). The excess material of the cervical region was removed with an exploratory probe, followed by photo-activation for 40 seconds. This was followed by occlusal adjustments and polishing of the restoration. Pulpectomy which was also planned for 75 and 85 was subsequently completed followed by stainless steel crown placed on 75. GIC restorations with Dycal (Dentsply, Milford, DE, USA) (Calcium hydroxide) base was placed on 54,64, and 74 . The treatment procedures were carried out in multiple visits accordingly. Fluoride application was done. Recommended oral hygiene instructions and diet counselling were given to parents. Recall visits for regular checkup to assess the oral status of the child was scheduled every 6 months (Figs. 6-8).

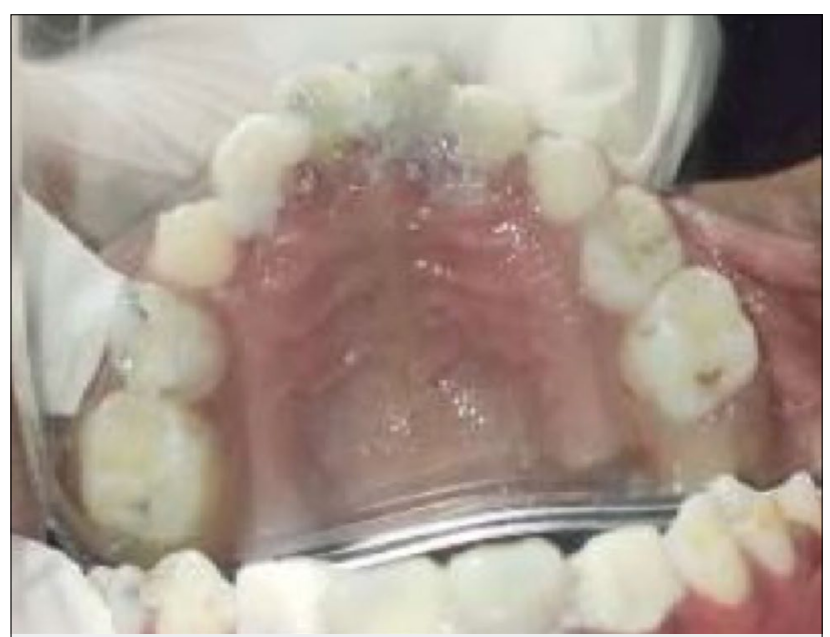

Figure 6. Postoperative photographs showing restored 51, 52, 54, 61 and 6264

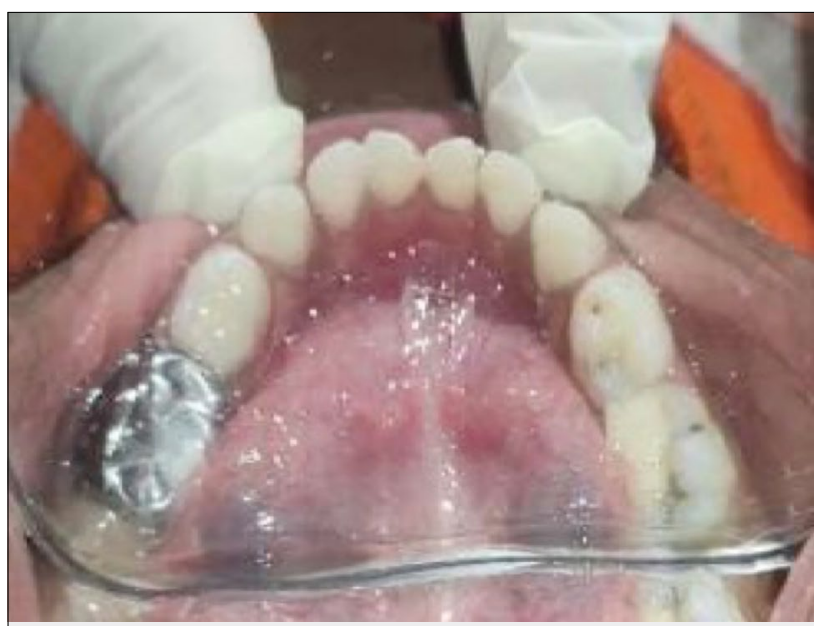

Figure 7. Postoperative photographs showing restored 74, 75 (stainless steel crown) and 85

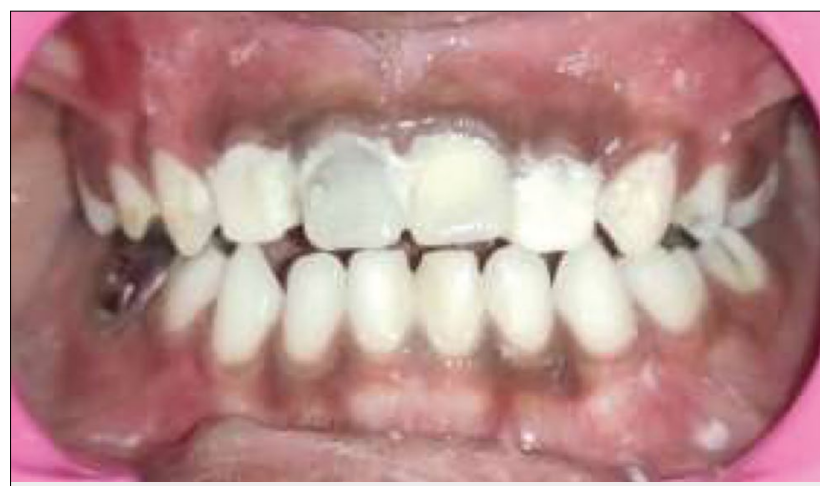

Figure 8. Postoperative photographs showing restored 74,75 (stainless steel crown) and 85 
The treatment procedures were carried out in multiple visits accordingly. Fluoride application was done. Recommended oral hygiene instructions and diet counselling were given to parents. Recall visits for regular checkup to assess the oral status of the child was scheduled every 6 months.

\section{Discussion}

Even with all the oral health prevention policies, dental caries remains one of the most prevalent diseases in preschool children resulting in the need for intervention by the dental professional at an earlier age. Primary teeth play a key role in the development of phonetics, eating, aesthetics and maintaining adequate space for permanent teeth in developing dentition.[5]

The etiology of ECC is a complex phenomenon and involves extensive interactions between social, behavioral and microbiological factors. [6] The available literature also confirms that dietary practices also contribute to ECC, specifically through the consumption of sugary drinks and a diet rich in sugars and other sweetening agents. [7] The oral rehabilitation of this case was based on the patient's age, cooperation during procedures and clinical findings accompanying the treatment plan. In addition to functional rehabilitation, esthetic rehabilitation in young children is also an important factor concerning esthetics and phonetics, as the absence of anterior teeth can cause the child to develop esthetic concerns and difficulty in pronunciation.

Premature loss or decay of primary teeth, especially primary anterior teeth will lead to the development of destructive habits such as tongue thrusting accompanied with subsidiary problems in speech and esthetic concerns leading to developing malocclusions and space loss which can hamper the normal growth and development of the child along with reduced selfesteem and other related psychological concerns. [8] The premature loss of anterior teeth results in an altered eruption path of succedaneous permanent teeth. This earlier loss results in increased arch length discrepancy, thereby acting as a contributory factor in developing malocclusion at a later stage. The age span of two to four years is considered to have specific importance because of ECC and/or trauma, the primary incisors get destructed fully or partially. [9] The lost at this age may result into psychological disturbances which will affect child's self-esteem and socialization, particularly if these defects are visible during speech.[10] In the present case, the child had grossly decayed anterior maxillary teeth, along with caries which were present on the occlusal surface of the posterior teeth of the maxilla and mandible. The grossly decayed anterior teeth were treated with pulp therapy followed by the placement of strip crowns, thereby preserving esthetics and formulating in assisting the child's developing phonetics.

The management of immature children had always been a serious concern for a pediatric dentist. The most preferred treatment strategy for such children is general anesthesia. However, due to the pertaining high costs of general anesthesia as well as the persisting complications and resultant hospital admission prevent majority of the parents from resorting to this option unless considered an emergency. One of the most important factors concerning the uncooperative behavior of the child is associated to child's fear and anxiety when they are being exposed to such treatment modalities. The management part included adequate behavior modification, restorative work, pulp therapy procedures, esthetic rehabilitation, recommended oral hygiene measures, and diet counseling with a follow-up every six months.

\section{Conclusion}

Early Childhood Caries is an important oral condition that requires prompt treatment and early intervention. Early diagnosis and awareness regarding ECC will help the child in leading a quality life. Composite restoration made with the aid of strip crowns provides a shorter clinical time, aesthetic result, re-establishment of the patient's smile and self-esteem were achieved. Appropriate diet modifications with adequate oral hygiene measures will certainly help in preventing ECC and thereby providing a healthy life to the children.

Financial Disclosure: Nil.

Conflict of Interest: None declared.

\section{References}

1. American Academy on Pediatric Dentistry; American Academy of Pediatrics. Policy on early childhood caries (ECC): classifications, consequences, and preventive strategies. Pediatr Dent 2008;30(7 Suppl):40-43

2. Colak H, Dülgergil CT, Dalli M, Hamidi MM. Early childhood caries update: A review of causes, diagnoses, and treatments. J Nat Sci Biol Med 2013;4(1):29-38 doi:10.4103/0976-9668.107257

3. Togoo RA. Early childhood caries-cause, diagnosis and management. IJHSR 2012;1(2):148-160 
4. Lenčová E, Pikhart H, Broukal Z. Early childhood caries trends and surveillance shortcomings in the Czech Republic. BMC Public Health 2012;12:547 doi:10.1186/1471-2458-12-547

5. Gussy MG, Waters EG, Walsh O, Kilpatrick NM. Early childhood caries: current evidence for aetiology and prevention. J Paediatr Child Health 2006;42(1-2):37-43 doi:10.1111/j.14401754.2006.00777.x

6. Feldens CA, Giugliani ER, Vigo Á, Vítolo MR. Early feeding practices and severe early childhood caries in four-year-old children from southern Brazil: a birth cohort study. Caries Res 2010;44(5):445-452 doi:10.1159/000319898

7. Cidro J, Zahayko L, Lawrence HP, Folster S, McGregor M, McKay K. Breast feeding practices as cultural interventions for early childhood caries in Cree communities. BMC Oral Health 2015;15:49 doi:10.1186/s12903-015-0027-5

8. Sharma N, Passi S, Kumar VV. Multidisciplinary approach to the rehabilitation in management of child with early childhood caries: a case report. J Clin Diagn Res 2013;7(10):2374-2375 doi:10.7860/JCDR/2013/5272.3528

9. Goenka P, Sarawgi A, Marwah N, Gumber P, Dutta S. Simple fixed functional space maintainer. Int J Clin Pediatr Dent 2014;7(3):225-228 doi:10.5005/jp-journals-10005-1272

10. Kanasi E, Dewhirst FE, Chalmers NI, et al. Clonal analysis of the microbiota of severe early childhood caries. Caries Res 2010;44(5):485-497 doi:10.1159/000320158 